\title{
Cytokines, paraoxonase-1, periostin and non-invasive liver fibrosis scores in patients with non-alcoholic fatty liver disease and persistently elevated aminotransferases: A pilot study
}

\author{
MIRCEA VASILE MILACIU ${ }^{1 *}$, LORENA CIUMĂRNEAN $^{1^{*}}$, DANIELA MARIA MATEI $^{2 *}$, ȘTEFAN CRISTIAN VESA $^{3}$, \\ OCTAVIA SABIN $^{3}$, IOANA CORINA BOCȘAN ${ }^{3}$, RALUCA MARIA POP ${ }^{3}$, VASILE NEGREAN ${ }^{1}$, \\ ANCA DANA BUZOIANU ${ }^{2}$ and MONICA ACALOVSCHI ${ }^{4}$
}

\begin{abstract}
${ }^{1}$ Department 5-Internal Medicine, 4th Medical Clinic, Faculty of Medicine, 'Iuliu Haţieganu’ University of Medicine and Pharmacy, 400015 Cluj-Napoca; ${ }^{2}$ Department 5-Internal Medicine, 3rd Medical Clinic, Faculty of Medicine, 'Iuliu Haţieganu' University of Medicine and Pharmacy, 400162 Cluj-Napoca; ${ }^{3}$ Department 2-Functional Sciences, Discipline of Pharmacology, Toxicology and Clinical Pharmacology, Faculty of Medicine, 'Iuliu Haţieganu' University of Medicine and Pharmacy, 400337 Cluj-Napoca; ${ }^{4}$ Doctoral School,

'Iuliu Haţieganu' University of Medicine and Pharmacy, 400012 Cluj-Napoca, Romania
\end{abstract}

Received October 26, 2020; Accepted November 25, 2020

DOI: 10.3892/etm.2021.9965

\begin{abstract}
Non-alcoholic fatty liver disease (NAFLD) is the most common liver disease worldwide. The aim of this study was to evaluate the possible association between paraoxonase-1 (PON1), periostin (POSTN), tumor necrosis factor (TNF)- $\alpha$, interleukin (IL)-6, IL-10 serum concentration with non-invasive liver fibrosis scores, in a cohort of patients with NAFLD. We studied a cohort of 52 patients diagnosed with NAFLD. The NAFLD fibrosis score (NFS), Fibrosis-4 Index (FIB-4), AST to platelet ratio index (APRI) and BARD scores were calculated for each patient. We determined the PON1, POSTN, TNF- $\alpha$, IL-6, and IL-10 serum values using ELISA kits. There was no correlation between PON1 or POSTN serum levels and non-invasive liver fibrosis. The TNF- $\alpha$ serum values were independently associated with the liver fibrosis scores $(\mathrm{P}=0.02$ for NFS and $\mathrm{P}=0.002$ for FIB-4). Age and metabolic syndrome were also independently linked to the fibrosis scores. In conclusion, serum levels of TNF- $\alpha$, age and metabolic syndrome were associated with the non-invasive liver fibrosis scores.
\end{abstract}

Correspondence to: Dr Ștefan Cristian Vesa, Department 2-Functional Sciences, Discipline of Pharmacology, Toxicology and Clinical Pharmacology, Faculty of Medicine, 'Iuliu Haţieganu' University of Medicine and Pharmacy, 23 Gheorghe Marinescu Street, 400337 Cluj-Napoca, Romania

E-mail: stefanvesa@gmail.com

*Contributed equally

Key words: non-alcoholic fatty liver disease, fibrosis scores, paraoxonase- 1 concentration, periostin, cytokines

\section{Introduction}

Non-alcoholic fatty liver disease (NAFLD) is the most common broad spectrum liver disease in developed countries. The potential evolution from simple steatosis (commonly referred as nonalcoholic fatty liver-NAFL) to steatohepatitis (NASH), advanced fibrosis, cirrhosis and, ultimately, hepatocellular carcinoma is one of the main reasons why NAFLD has gained much research attention in the last few years (1-3). A follow-up study by Ekstedt et al (4) addressed NASH as 'NAFLD and elevated liver enzymes'. Other follow-up studies showed that patients with NASH have a reduced survival compared to patients with simple steatosis $(4,5)$.

Hepatocyte damage induced by hepatic lipotoxicity is one of the main causes among the plethora of factors involved in NAFLD pathogenesis (6). Oxidative stress and endoplasmic reticulum stress can be triggered even as an adaptive response to lipotoxicity, which is a hepatic overflow of fatty acids, triglycerides, cholesterol, biliary acids and ceramides, among other active lipid metabolites (6-8). These lipids act as promoters of steatosis, reactive oxygen species (ROS) accumulation and alterators of liver signaling pathways (9). In addition, cytokine production and lipid peroxidation caused by ROS promote the progression of liver fibrosis and further hepatocellular injury $(7,10)$. It is currently accepted that an imbalance between anti-inflammatory cytokines [such as interleukin (IL)-10] and pro-inflammatory cytokines [IL-6 and tumor necrosis factor (TNF- $\alpha)$ ] could play an important role in the promotion of inflammation and progression of fibrosis in patients with NAFLD and particularly in NASH (10-12). High serum levels of TNF- $\alpha$ and IL-6 and low serum levels of IL-10 and adiponectin exert deleterious effects on NASH progression towards severe fibrosis $(6,7,13)$.

Liver fibrosis progression, independent of the presence of NASH, is the most crucial predictor of liver-related 
complications and overall mortality (14). Thus, it is important to establish an early diagnosis of advanced fibrosis in patients with NAFLD, likely using validated panels of serum biomarkers [e.g. Fibrosis-4 Index (FIB-4) or the NAFLD Fibrosis Score (NFS)] (15). In addition, transient elastography or newer techniques may be used to identify fibrosis, even if they overestimate the liver fibrosis in cases of severe steatosis (detected by ultrasonography or histology) (16).

Paraoxonase-1 (PON1) is an enzyme synthesized in the liver. PON1 exerts an important antioxidant, anti-inflammatory and anti-atherogenic effect by its main roles of protecting LDL-cholesterol from oxidation, reducing the transformation of macrophages into 'foam cells' and the catabolism of homocysteine thiolactone, among other activities $(17,18)$. PON1 activity is modulated by PON1 gene polymorphisms, but also by non-genetic factors (chemicals, drugs, smoking or diet, among others) (19). In chronic liver diseases (including NAFLD), PON1 activity is usually decreased, and this was found to be associated with alterations in HDL particles (20), peroxisome proliferator-activated receptor (PPAR) $\delta$ expression and upregulation of monocyte chemoattractant protein-1 (MCP-1) (21), together with an increase in TNF- $\alpha$ and IL-6 (22). All these associations suggest that low PON1 levels could be considered as a marker of lipid peroxidation, and a potential surrogate marker for enhanced oxidative stress and fibrosis in patients with NAFLD $(23,24)$.

Periostin (POSTN) is an extracellular matrix protein mainly secreted by osteoblasts, which exerts a pro-fibrotic effect in repairing damaged tissues (25). POSTN expression has been found to be associated with many diseases (including cancer), and recently it was suggested that this enzyme has a potential pro-fibrotic effect in the liver, mainly due to activation of lysyl-oxidase in hepatic stellate cells (HSCs) (26). Several studies have shown that serum POSTN levels are higher in patients with NAFLD compared to controls, but a potential causal relationship of POSTN and NAFLD has not been confirmed (25).

The aim of this pilot study was to evaluate PON1 and POSTN serum concentrations, together with the cytokine status (TNF- $\alpha$, IL-6, IL-10), in a cohort of patients with NAFLD and persistently elevated serum aminotransferases, and to correlate the findings with the liver fibrosis validated previously using non-invasive scores (FIB-4/NFS).

\section{Patients and methods}

The study was an observational, analytical, prospective, transversal and cohort type study. It was conducted at the Clinical CF University Hospital, Cluj-Napoca, Romania, between January 2016 and July 2019. The study was conducted according to the Declaration of Helsinki and was previously approved by the Ethics Committee of the 'Iuliu Hațieganu' University of Medicine and Pharmacy (no. 404/02/Jul/2015). All patients signed an informed consent form prior to study inclusion.

We consecutively enrolled 52 patients (mean age, 50 years; range, 18-70) diagnosed with NAFLD (either NAFL or NASH), with an equal distribution of men and women. Inclusion criteria included patients diagnosed with liver steatosis [by ultrasonography (US)] and moderately elevated aminotransferase levels at two or more prior visits and screened for a minimum of six months before study inclusion. All the enrolled subjects had negative biomarkers for viral hepatitis, autoimmune hepatitis, primary biliary cirrhosis or cholangitis, Wilson's disease or hemochromatosis. Liver cirrhosis or liver tumors were excluded clinically, biologically (normal coagulation parameters, normal albumin serum levels), ultrasonographically and by exclusion of portal hypertension (ultrasonographic signs, absence of splenomegaly and upper digestive endoscopy without gastroesophageal varices). Exclusion criteria consisted of significant chronic alcohol consumption, as defined as $\geq 30 \mathrm{~g} /$ day for men and $\geq 20 \mathrm{~g} /$ day for women (27); pregnancy; chronic use of medication with hepatotoxic potential and presence of any other disease proven to have an influence on POSTN and PON1 concentrations (active cancer or positive personal history of malignancy, asthma, thyroid gland dysfunctions, autoimmune disorders, psoriasis, allergies and psychiatric disorders).

The diagnosis of NAFLD was thus based on: i) The presence of liver steatosis evaluated by US; ii) exclusion of other liver conditions that may be evaluated with steatosis and persistently elevated aminotransferases; iii) exclusion of patients with significant alcohol consumption.

We recorded general information concerning each patient: Age, sex, body-mass index (BMI, calculated as the body mass divided by the square of the body height), and other comorbidities [pre-diabetes-impaired fasting glucose or/and impaired glucose tolerance, type 2 diabetes mellitus (T2DM) and metabolic syndrome]. A blood sample was obtained from each patient for routine assessments: Glycemia, aspartate aminotransferase (AST), alanine aminotransferase (ALT), alkaline phosphatase (ALP), $\gamma$-glutamyl transferase (GGT), platelet count (PLT), serum bilirubin, total cholesterol, HDL-cholesterol, triglycerides, and albumin. A separate blood sample was used for testing IL-6, IL-10, TNF- $\alpha$, highsensitivity C-reactive protein (Hs-CRP), PON1 and POSTN serum concentrations.

Routine laboratory testing was performed using different commercial kits for use with a Konelab Prime 60i analyzer (Thermo Fisher Scientific, Inc.). Hs-CRP, IL-6, IL-10 and TNF- $\alpha$ values were assessed using ELISA kits (Abbexa). POSTN and PON1 serum levels were determined by ELISA (Abbexa, USA) according to the manufacturer's instructions. For each assay, samples were diluted as needed and protein levels were calculated based on a four-parameter logistic (4-PL) curve-fit.

Abdominal ultrasound (US) was performed on each subject by the same experienced physician using a convex transducer on an Aloka Prosound Alpha 7 Premier ultrasound machine (Hitachi-Aloka Medical). Severity of steatosis was assessed by US, as described in detail in 2015 by Petta et al, and was established either as mild, moderate or severe NAFLD (16).

For each patient, we calculated the NAFLD fibrosis score (NFS), FIB-4 score, AST to platelet ratio index (APRI) and BARD score, using available free online calculators (WWw. mdcalc.com) and the NAFLD fibrosis score formula:

Formula $=1.675+0.037 \times$ age $($ years $)+0.094 \times$ BMI $\left(\mathrm{kg} / \mathrm{m}^{2}\right)+$ $1.13 \times$ IFG/diabetes $($ yes $=1$, no $=0)+0.99 \times$ AST/ALT ratio- 0.013 $\mathrm{x}$ platelets $\left(10^{9} / \mathrm{l}\right)-0.66 \mathrm{x}$ albumin $(\mathrm{g} / \mathrm{dl})$. 
For NFS, a low cutoff (lower than -1.455) excluded severe fibrosis, while a high score $(>0.676)$ was a predictor of severe fibrosis (28).

Statistical analysis. Statistical analysis was performed using MedCalc Statistical Software version 19.1.5 (MedCalc Software bv, Ostend, Belgium; https://www.medcalc.org; 2020). The quantitative data was tested for normality of the distribution (Shapiro Wilk test) and was characterized by median and $25-75$ percentiles. The qualitative variables were described by absolute and relative frequencies. Comparisons between groups were performed using the Man-Whitney or Kruskal-Wallis tests, whenever appropriate. Correlations between quantitative variables were verified using the Spearman's rank correlation coefficient. The independent association between variables and fibrosis scores was assessed by multivariate linear regression. The model included the variables that achieved a P-value $<0.2$ in the univariate analysis. A $\mathrm{P}$-value $<0.05$ was considered statistically significant.

\section{Results}

The clinical, biochemical and ultrasonographical data recorded for each subject are shown in Table I.

We did not find a statistically significant difference between sex in regards to the NFS $(\mathrm{P}=0.700), \mathrm{FIB}-4$ score $(\mathrm{P}=0.080)$, APRI score $(\mathrm{P}=0.200)$ and BARD score $(\mathrm{P}=0.800)$. The presence of metabolic syndrome was associated with significantly higher values for NFS $[-0.4(-1.6 ; 0)$ vs. $-2.78(-3.4 ;-2.1)$; $\mathrm{P}<0.001]$, FIB-4 score [1.5 $(0.9 ; 1.6)$ vs. $1.1(0.7 ; 1.5) ; \mathrm{P}=0.030]$, BARD score [2 $(2 ; 2)$ vs. $1(0.5 ; 1) ; \mathrm{P}<0.001]$. APRI score was not significantly higher in patients with metabolic syndrome $(\mathrm{P}=0.100)$.

The NFS and the FIB-4 score were strongly correlated with the age of the patients $(\mathrm{r}=0.501, \mathrm{P}<0.001$ and $\mathrm{r}=0.709, \mathrm{P}<0.001$, respectively), and moderately correlated with the IL-6 serum values $(r=0.336, P=0.010$ and $r=0.297, P=0.030$, respectively) (Table II). The NFS was moderately correlated with patient BMI and weakly correlated with the TNF- $\alpha$ serum levels. The BARD score was strongly correlated with patient BMI and weakly correlated with the IL-6 serum levels (Table II).

In order to evaluate the independent association of the clinical and laboratory variables with the fibrosis scores, we used multivariate linear regressions (Table III; Figs. 1 and 2). We obtained an $\mathrm{R}^{2}$ of 0.545 for the NFS, and $\mathrm{R}^{2}$ of 0.594 for the FIB- 4 score and an $\mathrm{R}^{2}$ of 0.489 for the BARD score. Age, metabolic syndrome and $\mathrm{TNF}-\alpha$ serum values were significantly correlated with the NFS. Furthermore, age and TNF- $\alpha$ serum values were independently linked to the FIB-4 score. The metabolic syndrome was the only independent variable significantly associated with the BARD score. The TNF- $\alpha$ was closely linked with the BARD score, but the statistical threshold was slightly passed.

\section{Discussion}

Non-alcoholic fatty liver disease (NAFLD) is a disease spectrum that is gaining more and more research interest, particularly due to its potential evolution from NAFL towards steatohepatitis (NASH), cirrhosis and hepatocellular
Table I. Descriptive variables of the study group.
Variables (unit of measurement; reference values)

Age (years) ${ }^{\mathrm{a}}$

BMI $\left(\mathrm{kg} / \mathrm{m}^{2}\right)^{\mathrm{a}}$

$\mathrm{T}^{2} \mathrm{DM}^{\mathrm{b}}$

Pre-diabetes ${ }^{\mathrm{b}}$

Metabolic syndrome ${ }^{\mathrm{b}}$

$\operatorname{AST}(U I / 1 ; 5-37)^{\mathrm{a}}$

$\operatorname{ALT}(\mathrm{UI} / 1 ; 5-40)^{\mathrm{a}}$

ALP (U/1; 98-279) ${ }^{\mathrm{a}}$

GGT (U/1; 7-32) ${ }^{\mathrm{a}}$

Total cholesterol (mg/dl; 100-200)

HDL-cholesterol (mg/dl; $>40)^{\mathrm{a}}$

Triglycerides (mg/dl; 45-140) ${ }^{\mathrm{a}}$

Total bilirubin $(\mathrm{mg} / \mathrm{dl} ; 0.3-1.2)^{\mathrm{a}}$

PLT $\left(10^{3} / \mu 1 ; 150-350\right)^{\mathrm{a}}$

Serum albumin $(\mathrm{g} / \mathrm{dl} ; 3.5-5.2)^{\mathrm{a}}$

Hs-CRP (ng/ml; 0.391-25) ${ }^{\mathrm{a}}$

IL-10 (pg/ml; 3.12-200) ${ }^{\mathrm{a}}$

IL-6 (pg/ml; 0.78-50) ${ }^{\mathrm{a}}$

TNF- $\alpha(\mathrm{pg} / \mathrm{ml} ; 15.6-1000)^{\mathrm{a}}$

POSTN (ng/ml; 7.8-500) ${ }^{\mathrm{a}}$

PON1 concentration (ng/ml; 3.12-200)

$\mathrm{NFS}^{\mathrm{a}}$

FIB-4 score ${ }^{\mathrm{a}}$

APRI score ${ }^{\mathrm{a}}$

BARD score
Patients with NAFLD $(\mathrm{N}=52)$

$50(37.25 ; 60.75)$

$30.23(27.49 ; 32.68)$

$12(23)$

15 (28.8)

$31(59.6)$

$52(45 ; 59)$

$70(62.25 ; 84.75)$

$215.5(171 ; 272.5)$

$38.5(32 ; 59)$

204.5 (175.25; 237)

$38(35 ; 50.75)$

$176.5(110 ; 211.75)$

$0.7(0.5 ; 0.87)$

$231(186 ; 268)$

$4.4(4.1 ; 4.9)$

$3.5(1.67 ; 5.2)$

$7.48(3.89 ; 9.06)$

$3.21(2.47 ; 4.41)$

$19.69(0 ; 95.5)$

$43.52(6.68 ; 114.55)$

$11.81(11.23 ; 12.37)$

$-1.55(-2.9 ;-0.33)$

$1.32(0.91 ; 2.04)$

$0.63(0.49 ; 0.88)$

$2(1 ; 2)$
Data are expressed as a median value/ 25 and 75 percentiles; ${ }^{\mathrm{b}}$ number of patients/percentage. NAFLD, non-alcoholic fatty liver disease; BMI, body mass index; T2DM, type 2 diabetes mellitus; AST, aspartate aminotransferase; ALT, alanine aminotransferase; GGT, $\gamma$-glutamyl transferase; PLT, platelet count; Hs-CRP, high-sensitivity C-reactive protein; IL, interleukin; TNF- $\alpha$, tumor necrosis factor- $\alpha$; POSTN, periostin; PON1, paraoxonase-1; NFS, NAFLD Fibrosis Score; FIB-4, Fibrosis-4 Index; APRI, AST to platelet ratio index.

carcinoma (1). As a specific treatment for NAFLD does not exist and the accuracy of liver biopsy for NASH diagnosis is not yet matched by other methods, current efforts are now focused on discovering non-invasive NAFL and/or NASH diagnostic scoring systems and targeted therapies $(1,29,30)$.

The concept of this study came from the pragmatic review of Dyson et al, which concluded that alanine aminotransferase (ALT) is a poor predictor of NAFLD presence, US is the first-line imaging technique and liver fat decreases as fibrosis increases (31). Thus, we designed a study which would include US evaluation of liver steatosis [as utilized by Petta et al (16)], validated non-invasive liver fibrosis markers (trying to replace the need for a liver biopsy) and serum assessment of potential biomarkers of liver impairment (either inflammation via oxidative stress [(PON1), cytokine activation (IL-6, TNF- $\alpha$ ) and promotion of fibrosis (POSTN)]. To our knowledge, this is the first study to evaluate a potential relation between PON1 
Table II. Correlations between fibrosis scores and clinical and biochemical markers of the study group.

\begin{tabular}{|c|c|c|c|c|c|c|c|c|}
\hline \multirow[b]{2}{*}{ Variables } & \multicolumn{2}{|c|}{ NFS } & \multicolumn{2}{|c|}{ FIB-4 score } & \multicolumn{2}{|c|}{ APRI score } & \multicolumn{2}{|c|}{ BARD score } \\
\hline & $\mathrm{r}$ & P-value & $\mathrm{r}$ & P-value & $\mathrm{r}$ & P-value & $\mathrm{r}$ & P-value \\
\hline Age (years) & 0.501 & $<0.001$ & 0.709 & $<0.001$ & 0.171 & 0.200 & 0.187 & 0.100 \\
\hline BMI $\left(\mathrm{kg} / \mathrm{m}^{2}\right)$ & 0.413 & 0.002 & 0.110 & 0.400 & 0.007 & 0.900 & 0.645 & $<0.001$ \\
\hline Hs-CRP (ng/ml) & 0.093 & 0.510 & 0.031 & 0.800 & 0.027 & 0.800 & 0.17 & 0.200 \\
\hline IL-10 (pg/ml) & 0.092 & 0.500 & 0.051 & 0.700 & 0.170 & 0.200 & 0.134 & 0.300 \\
\hline IL-6 (pg/ml) & 0.336 & 0.010 & 0.297 & 0.030 & 0.255 & 0.060 & 0.288 & 0.030 \\
\hline TNF- $\alpha(\mathrm{pg} / \mathrm{ml})$ & 0.266 & 0.050 & 0.226 & 0.100 & 0.155 & 0.270 & 0.178 & 0.200 \\
\hline POSTN (ng/ml) & -0.017 & 0.900 & -0.037 & 0.700 & 0.096 & 0.400 & -0.129 & 0.300 \\
\hline PON1 concentration $(\mathrm{ng} / \mathrm{ml})$ & 0.004 & 0.900 & 0.040 & 0.700 & 0.084 & 0.500 & 0.062 & 0.600 \\
\hline
\end{tabular}

$\mathrm{r}=$ correlation coefficient. BMI, body mass index; Hs-CRP, high-sensitivity C-reactive protein; IL, interleukin; TNF- $\alpha$, tumor necrosis factor- $\alpha$; POSTN, periostin; PON1, paraoxonase-1; NFS, NAFLD Fibrosis Score; FIB-4, Fibrosis-4 Index; APRI, AST to platelet ratio index.

Table III. Multivariate linear regressions for fibrosis scores.

\begin{tabular}{|c|c|c|c|c|c|c|}
\hline \multirow[b]{2}{*}{ Variables } & \multicolumn{2}{|c|}{ NFS } & \multicolumn{2}{|c|}{ FIB-4 score } & \multicolumn{2}{|c|}{ BARD score } \\
\hline & B & P-value & B & P-value & B & P-value \\
\hline Age (years) & 0.043 & 0.002 & 0.014 & $<0.001$ & 0.002 & 0.800 \\
\hline Metabolic syndrome & 1.593 & $<0.001$ & 0.097 & 0.080 & 1.062 & $<0.001$ \\
\hline IL-6 (pg/ml) & 0.126 & 0.200 & 0.080 & 0.400 & 0.066 & 0.200 \\
\hline TNF- $\alpha(\mathrm{pg} / \mathrm{ml})$ & 0.003 & 0.040 & 0.001 & 0.001 & 0.001 & 0.100 \\
\hline
\end{tabular}

$\mathrm{B}=$ unstandardized beta. IL, interleukin; TNF- $\alpha$, tumor necrosis factor- $\alpha$; NFS, NAFLD Fibrosis Score; FIB-4, Fibrosis-4 Index .

serum concentration and POSTN serum level and non-invasive liver fibrosis scores.

The median score for NFS in our patients was-1.55. This value is below the low cut-off score (-1.455) proposed by Angulo et al (28). The high cut-off value (>0.676), indicating a potentially advanced fibrosis, was found only in three patients. As for the FIB-4 score, which is considered to be one of the most useful and simple non-invasive tests to assess advanced fibrosis in NAFLD (32), the median score was 1.32. As it was proven that an FIB-4 score of $<1.3$ has a $90 \%$ negative predictive value (NPV) for advanced fibrosis, our cohort seemed to be a rather 'non-advanced liver fibrosis' one. The median APRI score was 0.63 in our study group, while the median BARD score was 2 . A recently published meta-analysis compared all the 4 scores used by us in a population of 13,046 patients with NAFLD based on 64 studies (33). The FIB-4 and NFS performed better than the others, both with NPV $>90 \%$ in ruling out advanced liver fibrosis (33), and have been lately proposed as first-line instruments for identifying patients that seem unlikely to need further assessment (34). A recently published study suggested a different approach (a step layered combination of non-invasive liver fibrosis markers to improve the accuracy of predicting advanced liver fibrosis), and showed that APRI, BARD, NIKEI (non-invasive Koeln-Essen-index) and FibroMeter NAFLD could be preferred to FIB-4 and NFS for the diagnosis of advanced fibrosis in NAFLD, due to a better diagnostic accuracy for liver fibrosis (35). In our study, the NAFLD fibrosis score was the only one to have multiple positive correlations with the other parameters. It was strongly correlated with patient age, moderately correlated with patient BMI and serum levels of IL-6, and weakly correlated with the serum values of TNF- $\alpha$.

We did not find a statistically significant correlation between PON1 serum concentration (which was rather low in our cohort, taking into consideration the detection range between 3.12-200 $\mathrm{ng} / \mathrm{dl}$ : Median=11.81 $\mathrm{ng} / \mathrm{dl}$ ) and the non-invasive fibrosis scores. Due to its protective effect against oxidative stress (36), we expected PON1 concentration to be correlated with the estimated degree of liver fibrosis in patients with NAFLD (especially in our cohort with persistently elevated aminotransferase levels). As we recently reported, PON1 serum concentration was found to be decreased in the serum of patients with NAFLD compared to subjects without NAFLD (37). In addition, in previous studies, we showed an association between PON1 and obesity and metabolic syndrome $(38,39)$. Still, in the present study, our cohort had a prevalence of metabolic syndrome of only $59.6 \%$, and this might partially explain the lack of correlation between PON1 serum levels and the non-invasive fibrosis scores. Another potential explanation of the lack of correlations between PON1 and fibrosis could have its origins in modulators of PON1. Activity of this enzyme is influenced by 


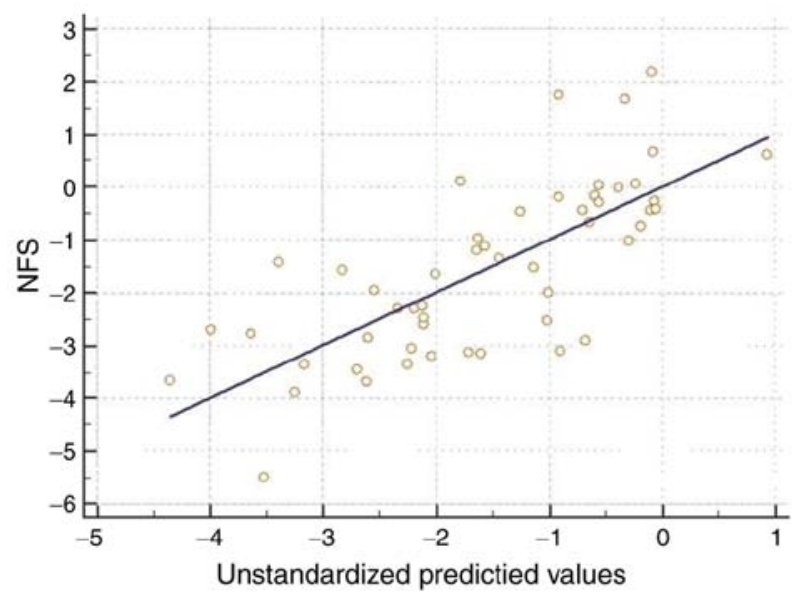

Figure 1. Multivariate linear regression plot for NFS. NFS, NFS, NAFLD Fibrosis Score.

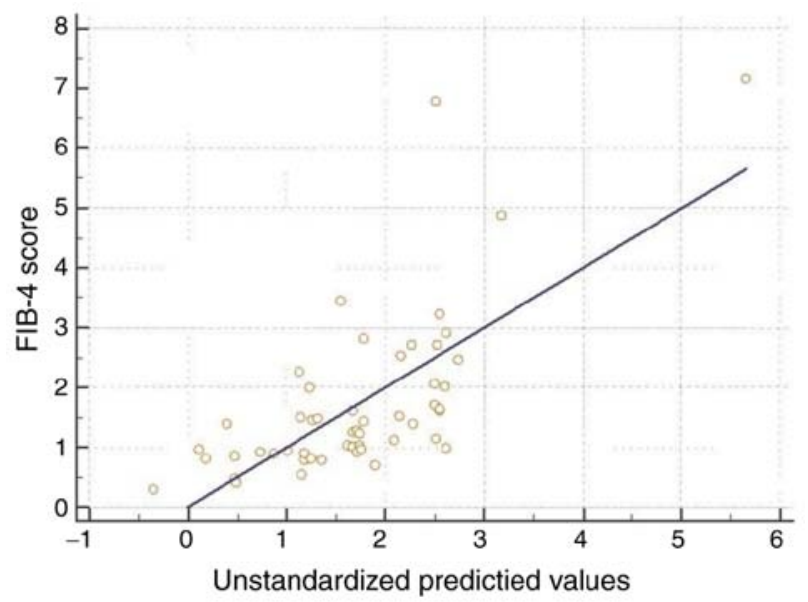

Figure 2. Multivariate linear regression plot for the FIB-4 score.FIB-4,Fibrosis-4 Index.

PON1 gene polymorphisms, and by other non-genetic factors. The L55M polymorphism seems to be associated with NAFLD (37), but its variants cannot fully explain the lack of correlation between PON1 and fibrosis in the present study. A recent study showed that PON1 activity is modulated only by resistin, among other cytokines and adipokines evaluated (IL-6, IL-8, TNF- $\alpha$, leptin, adiponectin) (40). Furthermore, it is currently accepted that high levels of peroxynitrite can Olead to modification of PON1 activity (41). This parameter could not be evaluated in our study. Another factor that was not evaluated in the present study was the diet of the subjects. As we previously mentioned, diet is an important modulator of PON1 activity. In the present study, it was difficult to record all of our patient dietary habits, although we observed that many of them were followers of a Western diet (known to have a negative impact on NAFLD). Finally, to the best of our knowledge, there is no published study which has evaluated the possible association between PON1 and liver fibrosis in NAFLD patients (either assessed by liver biopsy or by other methods such as fibrosis scores or imaging methods), thus the results of our pilot study cannot be compared with data from the literature.
As for POSTN, we also did not observe a statistically significant correlation between its serum levels and non-invasive fibrosis scores. In 2015, Amara et al showed that liver fibrogenesis is induced by TNF- $\alpha$ and IL-17, through enhanced expression of POSTN (42). Still, the main mechanism by which POSTN exerts its pro-fibrotic action in the liver is the activation of HSCs. POSTN was demonstrated to be at high levels in the serum of patients with cirrhosis, compared to controls, and at even higher values in patients with hepatocellular carcinoma (43). However, few subsequent clinical studies have suggested that NAFLD patients could benefit from treatment with POSTN antagonists and that POSTN could become a liver fibrosis biomarker in NAFLD (25). In our study, we found a lack of correlation between POSTN and non-invasive fibrosis scores, as, even if we did not find a similar study in the literature, we expected a direct relationship between this pro-fibrotic enzyme and NAFLD non-invasive fibrosis scores. A potential explanation for this lack of correlation comes from a study published after the writing of our study protocol. In that study, Takeda et al showed that POSTN cross-reacts with the renin-angiotensin system, and that blockade of the angiotensin-II receptor with losartan improved liver fibrosis (44). With many of our subjects being hypertensive and treated with angiotensin-II receptor blockers, the results may have been altered by this parameter.

In the multivariate linear regression model, we found that TNF- $\alpha$ was linked with the non-invasive fibrosis scores, mainly with FIB-4 and NFS. We also found an association between IL- 6 and the noninvasive fibrosis scores, an association which was not confirmed in the linear regression model. We then focused to find a potential explanation for the relationship between TNF- $\alpha$ and the non-invasive liver fibrosis scores in NAFLD. In a 4-year follow-up study, high serum levels of TNF- $\alpha$ were found to be associated with NAFLD development in subjects without NAFLD (45). In another study, TNF- $\alpha$ was associated with the likelihood of NAFLD presence, but also with high levels of IL-6 and visfatin, and decreased levels of adiponectin (46). In a population of pediatric patients with NAFLD, TNF- $\alpha$ serum levels were correlated with the histologic liver injury scores (47). Still, we were not able to identify a study that evaluated a potential direct relationship between serum TNF- $\alpha$ levels and non-invasive liver fibrosis scores, although TNF- $\alpha$ is known to play an important role in NAFLD, both in promoting steatosis and liver fibrosis $(48,49)$. This role is acknowledged mainly due to the enhancement of survival of HSCs, which was proven to be the main determinant of liver fibrosis (50). In addition, TNF- $\alpha$ promotes liver injury by enhancing hepatocyte apoptosis and activation of B cells, which further produce proinflammatory cytokines, mainly TNF- $\alpha$ and IL-6 (51). This is a potential explanation for the correlations between IL- 6 and the non-invasive fibrosis markers in our study (except for APRI score), even if a clear association was not found in the multivariate linear regressions. The usefulness of therapies with TNF- $\alpha$ antagonists (e.g. infliximab, adalimumab) is addressed by a recent review, which concludes that these agents may become useful medication for NASH (51).

The other two variables found to be associated with the non-invasive fibrosis scores were age and metabolic syndrome. Our result is consistent with the results of a recent study, in 
which the authors proved that the risk for severe fibrosis presented variability among age groups and that risk was higher in patients who presented more components of the metabolic syndrome (52).

Our study has several limitations. Firstly, the study included a moderate number of patients, mainly due to strict exclusion criteria. Secondly, although liver biopsy remains the definitive diagnosis of NAFLD and especially NASH, we were not able to perform it in all our patients, mainly due to their reluctance; also, we had to take into consideration its invasiveness and the sampling biases of this test (35). After exclusion of any other causes of liver impairment or significant alcohol consumption, in the context of persistently elevated aminotransferases, we supposed that our cohort might have been comprised of NASH patients (probably most of them). Still, we were unable to fully evaluate them (e.g. by using transient elastography or liver biopsy) because of method unavailability or because of patient reluctance to undergo an invasive test (like liver biopsy). Finally, because our subjects were recruited consecutively, the number of patients with presumed advanced liver fibrosis (as estimated by the non-invasive tests) was small.

Our study also has strengths. To the best of our knowledge, it is the first study which evaluates the association between PON1 and liver fibrosis, quantified by non-invasive fibrosis scores, in patients with NAFLD. Although our study sample is relatively small, the results showed that a correlation between PON1 or POSTN and non-invasive fibrosis scores is highly unlikely in patients with NAFLD, even within a larger study group.

Younossi et al suggested that, for the detection of advanced liver fibrosis, the existing biomarkers did not achieve the validity to be called 'surrogate endpoints' (32). Recent studies focus on finding non-invasive indexes useful in NAFLD (53). The further search for the ideal panel of biomarkers, able to distinguish 'benign' NAFL from NASH and cirrhosis, is the ultimate purpose of the focused efforts on NAFLD clinical research, because these markers could facilitate the finding of a pathogenetic treatment for this condition. In conclusion, serum levels of TNF- $\alpha$, age and metabolic syndrome, were associated with the non-invasive liver fibrosis scores. POSTN serum levels and PON-1 serum concentrations were not correlated with the non-invasive fibrosis scores. Thus, TNF- $\alpha$, age and the presence of metabolic syndrome might be considered for incorporation into a future comprehensive score for non-invasive evaluation of liver fibrosis in patients with NAFLD.

\section{Acknowledgements}

Not applicable.

\section{Funding}

This research study was partially financed by an internal grant (nos. 7690/72/15.04.2016 and 5200/62/01.03.2017) from the 'Iuliu Hațieganu' University of Medicine and Pharmacy Cluj-Napoca.

\section{Availability of data and materials}

The generated and analyzed data are included in this published article.

\section{Authors' contributions}

Conceptualization of the research study was carried out by MVM, ICB, LC, S,CV and MA. Methodology was achieved by MVM, LC, OS, ȘCV and MA and validation by MA, LC, DMM and VN. Formal analysis was conducted by ȘCV; investigation by MVM, LC, RMP, ICB and OS. Resources were accrued by ICB, ȘCV, RMP, DMM and ADB; writing-original draft preparation was carried out by MVM. Writing-review and editing was performed by S,CV, LC, ICB and MA; visualization by MVM; supervision by VN, ADB and MA. All authors read and approved the final version of the manuscript.

\section{Ethics approval and consent to participate}

The study was conducted according to the Declaration of Helsinki and was approved by the Ethics Committee of the 'Iuliu Hațieganu' University of Medicine and Pharmacy (no. 404/02/Jul/2015). All patients signed an informed consent form prior to study inclusion.

\section{Patient consent for publication}

Not applicable.

\section{Competing interests}

The authors declare no conflict of interest.

\section{References}

1. Sanyal AJ: Past, present and future perspectives in nonalcoholic fatty liver disease. Nat Rev Gastroenterol Hepatol 16: 377-386, 2019.

2. Friedman SL, Neuschwander-Tetri BA, Rinella M and Sanyal AJ: Mechanisms of NAFLD development and therapeutic strategies. Nat Med 24: 908-922, 2018.

3. European Association for the Study of the Liver (EASL); European Association for the Study of Diabetes (EASD); European Association for the Study of Obesity (EASO): EASL-EASD-EASO clinical practice guidelines for the management of non-alcoholic fatty liver disease. J Hepatol 64: 1388-1402, 2016.

4. Ekstedt M, Franzén LE, Mathiesen UL, Thorelius L, Holmqvist M, Bodemar $G$ and Kechagias S: Long-term follow-up of patients with NAFLD and elevated liver enzymes. Hepatology 44: 865-873, 2006.

5. Ekstedt M, Nasr P and Kechagias S: Natural history of NAFLD/NASH. Curr Hepatol Rep 16: 391-397, 2017.

6. Suzuki A and Diehl AM: Nonalcoholic steatohepatitis. Annu Rev Med 68: 85-98, 2017.

7. Yang J, Fernández-Galilea M, Martínez-Fernández L, González-Muniesa P, Pérez-Chávez A, Martínez JA and Moreno-Aliaga MJ: Oxidative stress and non-alcoholic fatty liver disease: Effects of omega-3 fatty acid supplementation. Nutrients 11: 872, 2019.

8. Mendez-Sanchez N, Cruz-Ramon VC, Ramirez-Perez OL, Hwang JP, Barranco-Fragoso B and Cordova-Gallardo J: New aspects of lipotoxicity in nonalcoholic steatohepatitis. Int J Mol Sci 19: 2034, 2018.

9. Kim KH and Lee MS: Pathogenesis of nonalcoholic steatohepatitis and hormone-based therapeutic approaches. Front Endocrinol (Lausanne) 9: 485, 2018.

10. Masarone M, Rosato V, Dallio M, Gravina AG, Aglitti A, Loguercio C, Federico A and Persico M: Role of oxidative stress in pathophysiology of nonalcoholic fatty liver disease. Oxid Med Cell Longev 2018: 9547613, 2018.

11. Bocsan IC, Milaciu MV, Pop RM, Vesa SC, Ciumarnean L, Matei DM and Buzoianu AD: Cytokines genotype-phenotype correlation in nonalcoholic steatohepatitis. Oxid Med Cell Longev 2017: 4297206, 2017. 
12. Tarantino G, Citro V and Capone D: Nonalcoholic fatty liver disease: A challenge from mechanisms to therapy. J Clin Med 9: $15,2019$.

13. Stojsavljević S, Gomerčić Palčić M, Virović Jukić L, Smirčić Duvnjak L and Duvnjak M: Adipokines and proinflammatory cytokines, the key mediators in the pathogenesis of nonalcoholic fatty liver disease. World J Gastroenterol 20: 18070-18091, 2014

14. Lindenmeyer CC and McCullough AJ: The natural history of nonalcoholic fatty liver disease-an evolving view. Clin Liver Dis 22: 11-21, 2018.

15. Stål P: Liver fibrosis in non-alcoholic fatty liver disease-diagnostic challenge with prognostic significance. World J Gastroenterol 21: 11077-11087, 2015.

16. Petta S, Maida M, Macaluso FS, Di Marco V, Camma C, Cabibi D and Crax1 A: The severity of steatosis influences liver stiffness measurement in patients with nonalcoholic fatty liver disease. Hepatology 62: 1101-1110, 2015.

17. Furlong CE, Marsillach J, Jarvik GP and Costa LG: Paraoxonases-1, -2 and -3 : What are their functions? Chem Biol Interact 259: 51-62, 2016

18. Shokri Y, Variji A, Nosrati M, Khonakdar-Tarsi A, Kianmehr A, Kashi Z, Bahar A, Bagheri A and Mahrooz A: Importance of paraoxonase 1 (PON1) as an antioxidant and antiatherogenic enzyme in the cardiovascular complications of type 2 diabetes: Genotypic and phenotypic evaluation. Diabetes Res Clin Pract 161: 108067, 2020.

19. Ciumarnean L, Milaciu MV, Macarie AE, Sampelean DP and Achimas-Cadariu A: Non-genetic factors influencing serum PON1 levels. HVM Bioflux 6: 20-24, 2014

20. Marsillach J, Aragonès G, Mackness B, Mackness M, Rull A, Beltrán-Debón R, Pedro-Botet J, Alonso-Villaverde C, Joven J and Camps J: Decreased paraoxonase-1 activity is associated with alterations of high-density lipoprotein particles in chronic liver impairment. Lipids Health Dis 9: 46, 2010.

21. Marsillach J, Camps J, Ferre N, Beltran R, Rull A, Mackness B, Mackness M and Joven J: Paraoxonase-1 is related to inflammation, fibrosis and PPAR delta in experimental liver disease. BMC Gastroenterol 9: 3, 2009.

22. Fuhrman B: Regulation of Hepatic Paraoxonase-1 expression. J Lipids 2012: 684010, 2012.

23. Samy W and Hassanian MA: Paraoxonase-1 activity, malondialdehyde and glutathione peroxidase in non-alcoholic fatty liver disease and the effect of atorvastatin. Arab J Gastroenterol 12: $80-85,2011$.

24. Lou-Bonafonte JM, Gabás-Rivera C, Navarro MA and Osada J: PON1 and mediterranean diet. Nutrients 7: 4068-4092, 2015.

25. Jia Y, Zhong F, Jiang S, Guo Q, Jin H, Wang F, Li M, Wang L, Chen A, Zhang F, et al: Periostin in chronic liver diseases: Current research and future perspectives. Life Sci 226: 91-97, 2019.

26. Kumar P, Smith T, Raeman R, Chopyk DM, Brink H, Liu Y, Sulchek T and Anania FA: Periostin promotes liver fibrogenesis by activating lysyl oxidase in hepatic stellate cells. J Biol Chem 293: 12781-12792, 2018

27. Ratziu V, Bellentani S, Cortez-Pinto H, Day C and Marchesini G: A position statement on NAFLD/NASH based on the EASL 2009 special conference. J Hepatol 53: 372-384, 2010.

28. Angulo P, Hui JM, Marchesini G, Bugianesi E, George J, Farrell GC, Enders F, Saksena S, Burt AD, Bida JP, et al: The NAFLD fibrosis score: A noninvasive system that identifies liver fibrosis in patients with NAFLD. Hepatology 45: 846-854, 2007.

29. Drescher HK, Weiskirchen S and Weiskirchen R: Current status in testing for nonalcoholic fatty liver disease (NAFLD) and nonalcoholic steatohepatitis (NASH). Cells 8: 845, 2019.

30. Ratziu V: A critical review of endpoints for non-cirrhotic NASH therapeutic trials. J Hepatol 68: 353-361, 2018.

31. Dyson JK, Anstee QM and McPherson S: Non-alcoholic fatty liver disease: A practical approach to diagnosis and staging. Frontline Gastroenterol 5: 211-218, 2014.

32. Younossi ZM, Loomba R, Anstee QM, Rinella ME, Bugianesi E, Marchesini G, Neuschwander-Tetri BA, Serfaty L, Negro F, Caldwell SH, et al: Diagnostic modalities for nonalcoholic fatty liver disease, nonalcoholic steatohepatitis, and associated fibrosis. Hepatology 68: 349-360, 2018.

33. Xiao G, Zhu S, Xiao X, Yan L, Yang J and Wu G: Comparison of laboratory tests, ultrasound, or magnetic resonance elastography to detect fibrosis in patients with nonalcoholic fatty liver disease: A meta-analysis. Hepatology 66: 1486-1501, 2017.

34. Castera L, Friedrich-Rust M and Loomba R: Noninvasive assessment of liver disease in patients with nonalcoholic fatty liver disease. Gastroenterology 156: 1264-1281.e4, 2019.
35. Yang M, Jiang L, Wang Y, Li X, Zou Z, Han T, Nan Y, Lu F and Zhao J: Step layered combination of noninvasive fibrosis models improves diagnostic accuracy of advanced fibrosis in nonalcoholic fatty liver disease. J Gastrointestin Liver Dis 28: 289-296, 2019.

36. Levy D, Reichert CO and Bydlowski SP: Paraoxonases activities and polymorphisms in elderly and old-age diseases: An overview. Antioxidants (Basel) 8: 118, 2019.

37. Milaciu MV, Vesa SC, Bocșan IC, Ciumărnean L, Sâmpelean D, Negrean V, Pop RM̉, Matei DM, Pașca S, Răchișan AL, et al: Paraoxonase-1 serum concentration and PON1 gene polymorphisms: Relationship with non-alcoholic fatty liver disease. J Clin Med 8: 2200, 2019.

38. Ciumarnean L, Vesa SC, Dronca E, Sampelean DP, Vlad VC, Moldovan MS and Achimas CA: Distribution of paraoxonase 1 polymorphisms and activities in obese patients. Rev Romana Med Lab 21: 381-389, 2013.

39. Ciumarnean L, Dronca E, Vesa SC, Sampelean D, Buzoianu AD and Achimas-Cadariu A: Paraoxonase 1 genotype-phenotype correlation in patients with metabolic syndrome. Rom J Morphol Embryol 56: 387-392, 2015.

40. Tisato V, Romani A, Tavanti E, Melloni E, Milani D, Bonaccorsi G, Sanz JM, Gemmati D, Passaro A and Cervellati C: Crosstalk between adipokines and paraoxonase 1: A new potential axis linking oxidative stress and inflammation. Antioxidants (Basel) 8: 287, 2019.

41. Marín M, Moya $C$ and Máñez S: Mutual influences between nitric oxide and paraoxonase 1. Antioxidants (Basel) 8: 619, 2019

42. Amara S, Lopez K, Banan B, Brown SK, Whalen M, Myles E, Ivy MT, Johnson T, Schey KL and Tiriveedhi V: Synergistic effect of pro-inflammatory $\mathrm{TNF} \alpha$ and IL-17 in periostin mediated collagen deposition: Potential role in liver fibrosis. Mol Immunol 64: 26-35, 2015

43. Lv Y, Wang W, Jia WD, Sun QK, Huang M, Zhou HC, Xia HH, Liu WB, Chen H, Sun SN and Xu GL: High preoparative levels of serum periostin are associated with poor prognosis in patients with hepatocellular carcinoma after hepatectomy. Eur J Surg Oncol 39: 1129-1135, 2013.

44. Takeda K, Noguchi R, Kitade M, Namisaki T, Moriya K, Kawaratani H, Okura Y, Kaji K, Aihara Y, Douhara A, et al: Periostin cross-reacts with the renin-angiotensin system during liver fibrosis development. Mol Med Rep 16: 5752-5758, 2017

45. Seo YY, Cho YK, Bae JC, Seo MH, Park SE, Rhee EJ, Park CY, Oh KW, Park SW and Lee WY: Tumor necrosis factor- $\alpha$ as a predictor for the development of nonalcoholic fatty liver disease: A 4-year follow-up study. Endocrinol Metab (Seoul) 28: 41-45, 2013.

46. Jamali R, Arj A, Razavizade M and Aarabi MH: Prediction of nonalcoholic fatty liver disease via a novel panel of serum adipokines. Medicine (Baltimore) 95: e2630, 2016.

47. Manco M, Marcellini M, Giannone G and Nobili V: Correlation of serum TNF-alpha levels and histologic liver injury scores in pediatric nonalcoholic fatty liver disease. Am J Clin Pathol 127: 954-960, 2007.

48. Niederreiter L and Tilg H: Cytokines and fatty liver disease. Liv Res 2: 14-20, 2018.

49. Copaci I, Micu L and Voiculescu M: The role of cytokines in non-alcoholic steatohepatitis. A review. J Gastrointestin Liv Dis 15: 363-373, 2006.

50. Yang YM and Seki E: TNF $\alpha$ in liver fibrosis. Curr Pathobiol Rep 3: 253-261, 2015.

51. Lopetuso LR, Mocci G, Marzo M, D'Aversa F, Rapaccini GL, Guidi L, Armuzzi A, Gasbarrini A and Papa A: Harmful effects and potential benefits of anti-tumor necrosis factor (TNF)- $\alpha$ on the liver. Int J Mol Sci 19: 2199, 2018.

52. Petta S, Eslam M, Valenti L, Bugianesi E, Barbara M, Camma C, Porzio M, Rosso C, Fargion S, George J and Craxì A: Metabolic syndrome and severity of fibrosis in nonalcoholic fatty liver disease: An age-dependent risk profiling study. Liver Int 37: 1389-1396, 2017.

53. Culafic M, Vezmar Kovacevic S, Dopsaj V, Stulic M, Vlaisavljevic Z, Miljkovic B and Culafic D: A simple index for nonalcoholic steatohepatitis-HUFA-Based on routinely performed blood tests. Medicina (Kaunas) 55: 243, 2019.

This work is licensed under a Creative Commons Attribution-NonCommercial-NoDerivatives 4.0 International (CC BY-NC-ND 4.0) License. 